\title{
Development and experimental validation of a versatile prototype Swing Arm Profilometer for measuring optical surfaces
}

\author{
Hongwei Jing \\ hwjing@ioe.ac.cn \\ Zhaoheng Lin \\ Lifang Ma
}

Shibin Wu

Fan Wu

\author{
Institute of Optics and Electronics, Chinese Academy of Sciences, P.O .Box 350, Chengdu, 610209, China \\ Institute of Optics and Electronics, Chinese Academy of Sciences, P.O .Box 350, Chengdu, 610209, China \\ Institute of Optics and Electronics, Chinese Academy of Sciences, P.O .Box 350, Chengdu, 610209, China
}

Institute of Optics and Electronics, Chinese Academy of Sciences, P.O .Box 350, Chengdu, 610209, China

Institute of Optics and Electronics, Chinese Academy of Sciences, P.O .Box 350, Chengdu, 610209, China

\begin{abstract}
A versatile prototype Swing Arm Profilometer (SAP) is developed and experimentally validated in the Institute of Optics and Electronics (IOE), Chinese Academy of Sciences (CAS). The configurations for the SAP are introduced. The measurement principles for measuring plano, spherical and aspherical mirrors are given. Four experiments are performed on the prototype SAP and validated by interferometric results. The measurement uncertainty for the current prototype SAP is 0.5 micron (rms). [DOI: http://dx.doi.org/10.2971/jeos.2011.11052]
\end{abstract}

Keywords: development, experimental validation, swing arm profilometer, measurement

\section{INTRODUCTION}

With the fast development of technologies, the optical surfaces are widely used in high technology areas such as astronomy, space optics, nucleus fusion and laser beam systems. At the same time, the apertures of these optical surfaces are becoming larger and larger, the F-numbers are becoming smaller and smaller. This is a great challenge to the traditional optical fabrication and testing. The process of the fabrication can be subdivided into three stages: the kibbling process, the grinding process and the polishing process.

The testing technologies in polishing process are well studied [1], the standard instrument is interferometer. But the interferometer has small measurement range. The surface error should be small enough to be tested by the interferometer.

The testing technologies in kibbling and grinding process are as important as the polishing process. During this process, the surface error will converge from tens to microns to submicrons. Traditionally the coordinate measurement devices are employed to test the optical surfaces, such as Coordinates Measurement Machines and bridge profilometers [2]-[3].But these devices need the optical surfaces under test to be moved to the measurement volume. Its time-consuming and sometimes dangerous.

In recent 20 years, the SAP technology is developed in the University of Arizona and University College London. And they have reported outstanding results [4]-[11].The SAP is fit for plano, spherical and aspherical mirrors, even for the off-axis aspherical mirrors during the grinding and prepolishing process. Besides, the SAP can be mounted besides the grinding machine and do in-situ measurements.

In this paper, we report the development and experimental validation of a versatile prototype Swing Arm Profilometer (SAP) in the Institute of Optics and Electronics (IOE), Chinese Academy of Sciences (CAS). The paper is arranged as follows. In Section 2, the configurations of the SAP are described. In Section 3, a detailed swing arm profilometry principle is provided and 4 validation experiments are presented. In Section 4 , measurement uncertainty for the prototype SAP is reported, leading to concluding remarks in Section 5.

\section{Configurations of the SAP}

The prototype SAP developed in the IOE, CAS principally comprises a mirror bearing supporting the surface under test (SUT), a pivoted swinging arm that swings across the SUT, an arm bearing that rotates the probe and counter balance for the arm. The basic configurations of the SAP are shown in Fig. 1.

The current prototype SAP was built using the existing parts in IOE, CAS. The mirror bearing is the bearing of a 


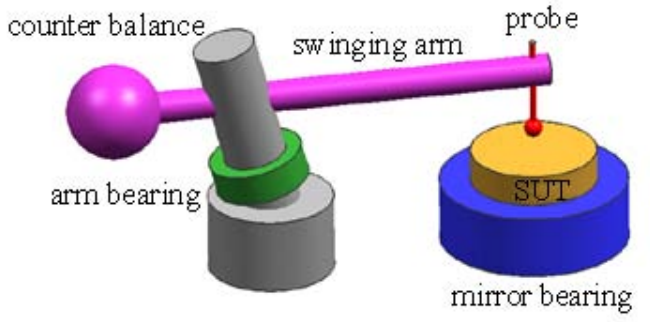

FIG. 1 Configurations of the prototype SAP

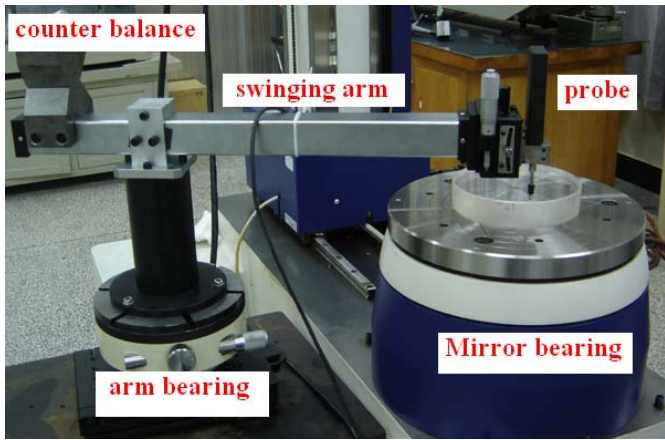

FIG. 2 The practical configurations of the prototype SAP

cylindricity measurement system, with $20 \mathrm{~nm}$ in axial runout and 0.06 arc second in tilt error. The mirror bearing is made of steel with $11.7 \mathrm{ppm} / \mathrm{K}$ in Coefficient of Temperature Expansion (CTE). The probe is a Heidenhain MT2571 linear encoder, with 0.2 microns accuracy and $50 \mathrm{~nm}$ resolution. The swinging arm and the counter balance are manufactured in IOE, CAS, which are made of steel with $11.7 \mathrm{ppm} / \mathrm{K}$ in CTE. The arm bearing is a product from IOE, CAS, with 0.5 microns in axial runout and 0.5 arc second in tilt error. The arm bearing is made of steel with $11.7 \mathrm{ppm} / \mathrm{K}$ in CTE. The experimental SUTs are made of BK7 glass with $7.5 \mathrm{ppm} / \mathrm{K}$ in CTE.

The practical configurations of the prototype SAP are shown in Fig 2.

\section{Measurement principle and experimental results}

The prototype SAP is a versatile measurement instrument for measuring the surface error of the mirrors [4]-[7]. Which can measure plano, spherical and aspherical mirrors with different instrument setup.

As shown in Fig. 3, the SAP is read for measuring the plano mirrors by adjusting the rotary axis of the arm bearing and the rotary axis of the mirror bearing to be parallel. As shown in Fig. 4, the SAP is read for measuring the concave spherical

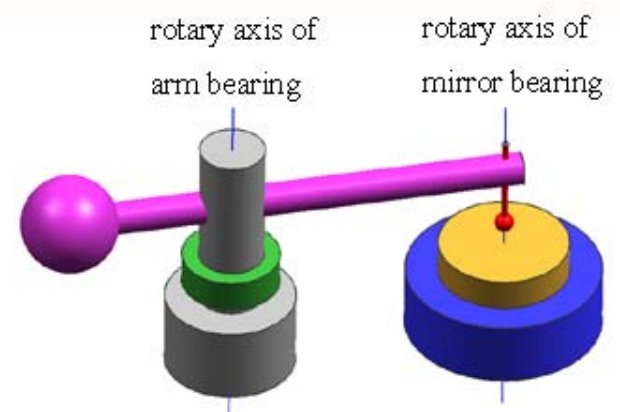

FIG. 3 Measurement setup for plano surface

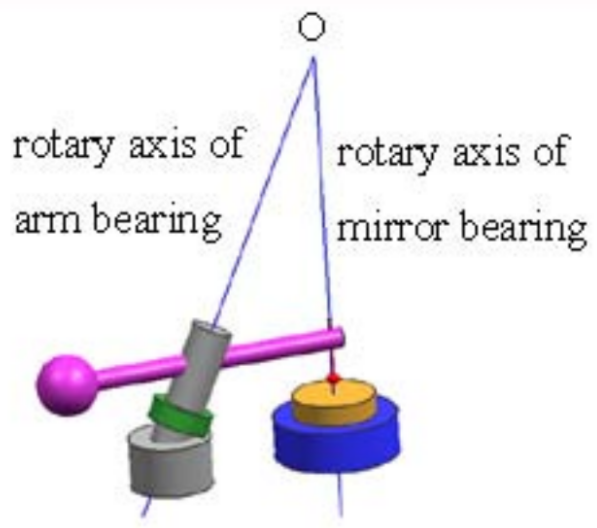

FIG. 4 Measurement setup for concave spherical surface

mirrors by adjusting the rotary axis of the arm bearing and the rotary axis of the mirror bearing to intersect at $\mathrm{O}$ point. The $\mathrm{O}$ point is the center of the spherical mirror under test. As shown in Fig. 5, the SAP is read for measuring the convex spherical mirrors by adjusting the rotary axis of the arm bearing and the rotary axis of the mirror bearing to intersect at $\mathrm{O}$ point. The $\mathrm{O}$ point is the center of the spherical mirror under test.

When measuring aspherical mirrors, the instrument setup is the same as the measurement of the spherical mirrors.

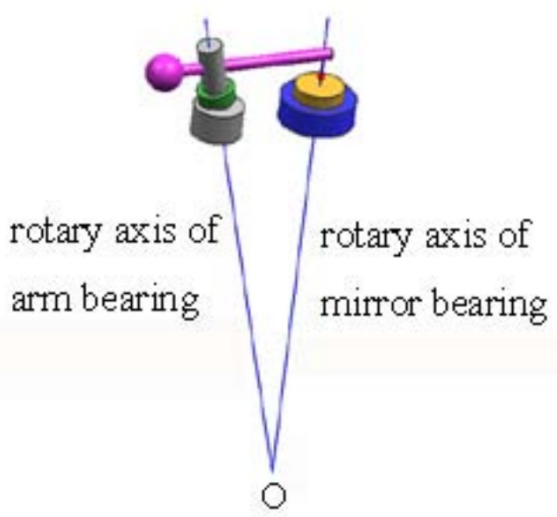

FIG. 5 Measurement setup for convex spherical surface 


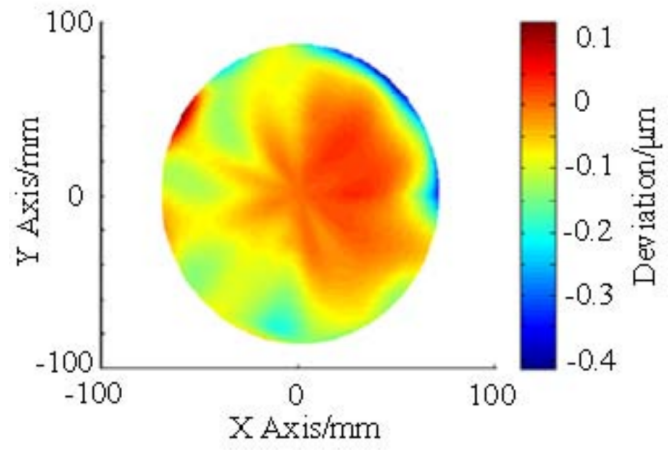

FIG. 6 SAP results for plano mirror $(\mathrm{PV}=0.5 \mu \mathrm{m}, \mathrm{rms}=0.1 \mu \mathrm{m})$

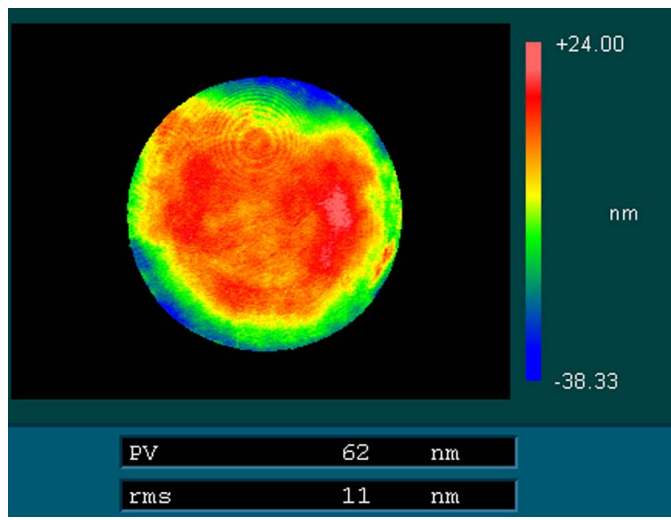

FIG. 7 Interferometric result for plano mirror $(\mathrm{PV}=0.062 \mu \mathrm{m}, \mathrm{rms}=0.011 \mu \mathrm{m})$

To validate the measurement accuracy of the prototype SAP. Four validation experiments are performed in our metrology lab with temperature control of $20^{\circ} \mathrm{C} \pm 0.5^{\circ} \mathrm{C}$, the fluctuation of temperature is $0.5{ }^{\circ} \mathrm{C} /$ day. The measurement device is placed on an isolated cement base.

Firstly, a plano standard mirror with $150 \mathrm{~mm}$ in diameter is measured using the prototype SAP. The result of the SAP measurement is shown in Fig. 6. The validation interferometric result is shown in Fig. 7. Secondly, a concave spherical mirror standard ( $R=5757.5 \mathrm{~mm}, 100 \mathrm{~mm}$ in diameter $)$ is measured using the prototype SAP. The result of the SAP measurement is shown in Fig. 8. The validation interferometric result is shown in Fig. 9. Thirdly, a convex spherical mirror $(R=5755.7 \mathrm{~mm}$, $100 \mathrm{~mm}$ in diameter) is measured using the prototype SAP. The result of the SAP measurement is shown in Fig. 10. The vali-

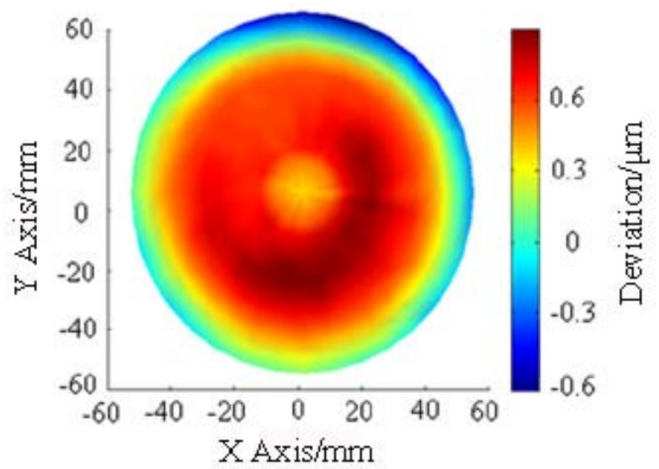

FIG. 8 SAP results for concave spherical mirror $(\mathrm{PV}=1.5 \mu \mathrm{m}$ rms $=0.3 \mu \mathrm{m})$

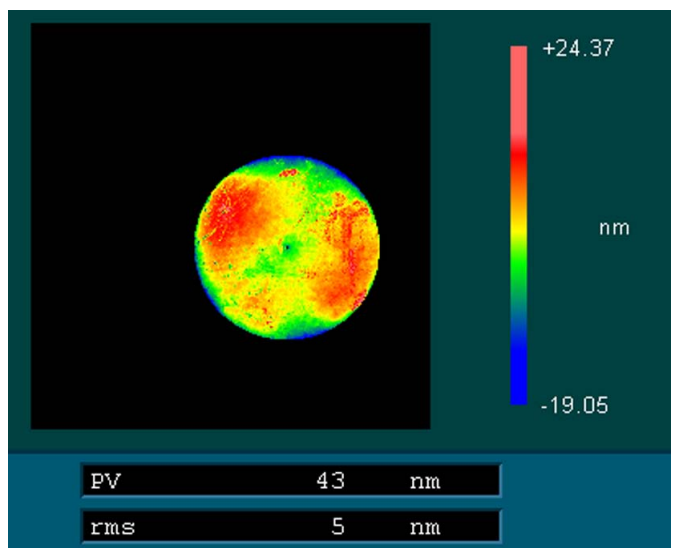

FIG. 9 Interferometric result for concave spherical mirror $(\mathrm{PV}=0.043 \mu \mathrm{m}, \mathrm{rms}=0.005$ $\mu \mathrm{m})$

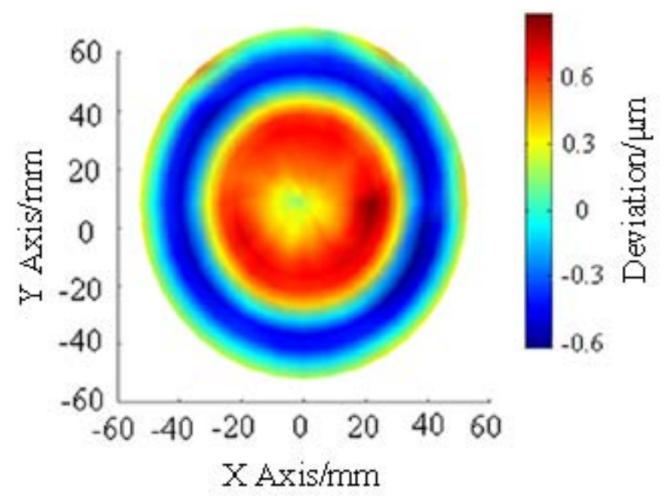

FIG. 10 SAP results for concave spherical mirror $(\mathrm{PV}=1.7 \mu \mathrm{m}, \mathrm{rms}=0.4 \mu \mathrm{m})$

dation interferometric result is shown in Fig 11. Fourthly, a parabolic mirror ( $F=520 \mathrm{~mm}, 200 \mathrm{~mm}$ in diameter) is measured using the prototype SAP. The result of the SAP measurement is shown in Fig. 12. The validation interferometric result is shown in Fig. 13. The validation measurement is done by null test using an interferometer and a mirror flat (with a 80 $\mathrm{mm}$ hole in the middle).

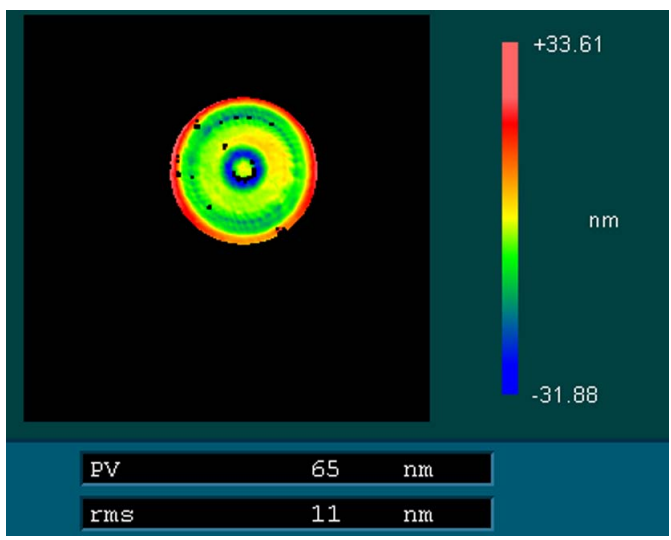

FIG. 11 Interferometric result for concave spherical mirror $(\mathrm{PV}=0.065 \mu \mathrm{m}, \mathrm{rms}=0.011$ $\mu \mathrm{m})$ 


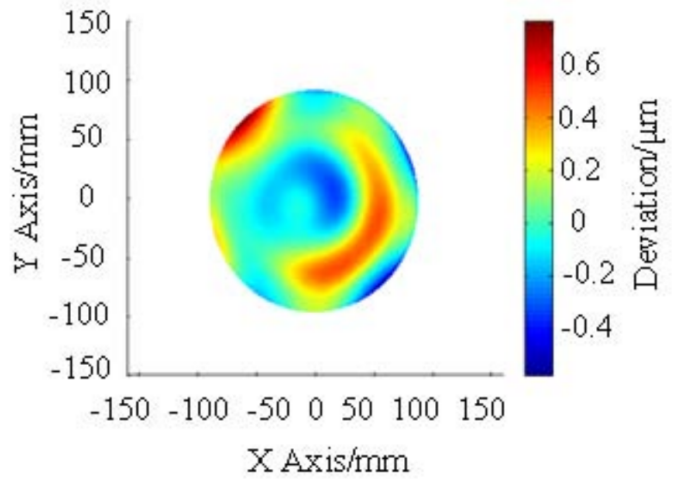

FIC. 12 SAP results for parabolic mirror $(\mathrm{PV}=1.3 \mu \mathrm{m}, \mathrm{rms}=0.3 \mu \mathrm{m})$

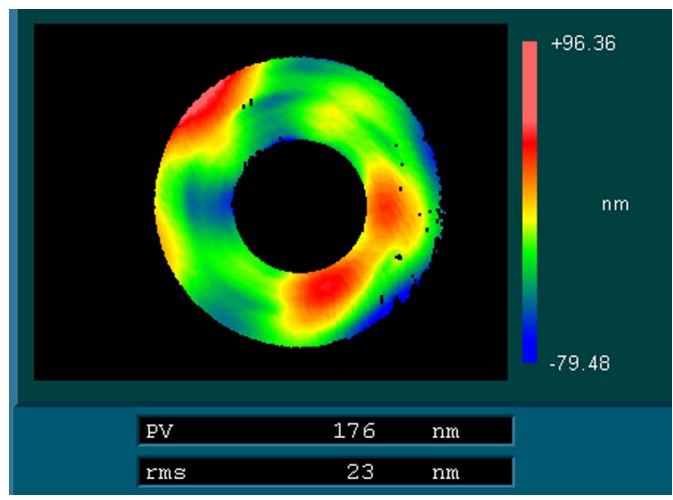

FIG. 13 Interferometric result for parabolic mirror $(\mathrm{PV}=0.176 \mu \mathrm{m}, \mathrm{rms}=0.023 \mu \mathrm{m})$

\section{Measurement uncertainty analysis}

Because the surface error parameters (PV and rms) are calculated using $\mathrm{Z}$ coordinates (vertical direction). The uncertainty analysis is based on contributions in vertical direction.

The contributions of the uncertainty comes from the axial runout of the arm bearing, tilt error of the arm bearing, axial runout of the mirror bearing, the probe, the CTE difference between segments, long time stability of the system and vibration isolation.

According to the specifications of the arm bearing, the axial runout of the arm bearing is 0.5 micron. The measurement uncertainty contribution from the axial runout of the arm bearing is

$$
u_{1}=0.5 \mu \mathrm{m}
$$

According to the specifications of the arm bearing, the tilt error of the arm bearing is 0.5 arc second. For a $450 \mathrm{~mm}$ arm, the measurement uncertainty contribution from the tilt error of the arm bearing is

$$
u_{2}=\tan \left(\frac{0.5}{3600}\right) \times 450=1.1 \mu \mathrm{m}
$$

According to the specifications of the mirror bearing, the axial runout of the mirror bearing is $20 \mathrm{~nm}$. The measurement uncertainty contribution from the axial runout of the mirror bearing is

$$
u_{3}=0.02 \mu \mathrm{m}
$$

According to the specifications of the probe, the measurement uncertainty contribution from the probe is

$$
u_{4}=0.2 \mu \mathrm{m}
$$

The CTE difference between mechanical parts and the BK7 glass is $4.2 \mathrm{ppm} / \mathrm{K}$, the displacement between table top of the mirror bearing and probe is $50 \mathrm{~mm}$. For $0.5^{\circ} \mathrm{C}$ temperature change, the measurement uncertainty contribution is

$$
u_{5}=4.2 \times 0.05 \times 0.5=0.1 \mu \mathrm{m}
$$

To determine the long time stability of the system, two calibration experiments are performed using the same measurement setup. The time span between the two experiments is 8 hours. The results difference is 0.2 micron. The measurement uncertainty contribution from the long time stability of the system is

$$
u_{6}=0.2 \mu \mathrm{m}
$$

The prototype SAP is mounted in the metrology lab and placed on an isolated cement base. The contribution from the vibration is considered negligible. The combined uncertainty is

$$
u_{c}=\sqrt{u_{1}^{2}+u_{2}^{2}+u_{3}^{2}+u_{4}^{2}+u_{5}^{2}+u_{6}^{2}}=1.25 \mu \mathrm{m}
$$

The expanded uncertainty $(\mathrm{k}=2)$ is

$$
U_{P V}=k \times u_{c}=2.5 \mu \mathrm{m}
$$

This is the measurement uncertainty for PV value. According the measurement experience, the measurement uncertainty of rms value is about one fifth of the PV value, which is

$$
U_{r m s}=\frac{U_{P V}}{5}=0.5 \mu \mathrm{m}
$$

\section{Conclusions and Discussions}

In this paper, we presented the development and experimental validation of a versatile prototype SAP in IOE, CAS. The configurations for the SAP together with the measurement principles for measuring plano, spherical and aspherical mirrors are introduced. Four plano, spherical and aspherical mirror samples are measured on the prototype SAP. The measurement uncertainty for the current prototype SAP is 0.5 micron (rms).

Comparing the SAP results with the interferometric results, it is clear the SAP results show good agreement with the interferometric results and the differences between the two measurement methods are within the measurement uncertainty. This proves the measurement results are valid, the prototype SAP has the ability to measure surface error to 0.5 micron (rms).

The current prototype SAPs main error comes from the arm bearing. To measure the optical surfaces more precisely, better arm bearing should be used. The work in this paper is only a prelude for the SAP development in IOE, CAS. The SAP with better bearings and non-contact probe is under development. The prototype SAP is being used in the workshop for measuring an off-axis parabolic mirror [12]. More research papers will be published in future. 


\section{Acknowledgements}

The authors acknowledge support from the National Natural Science Foundation of China (NSFC), under Grant No.60838002 and Grant No.60908042. The authors gratefully thank Lin Luo for manufacturing the parabolic mirror.

\section{References}

[1] D. Malacara, Optical Shop Testing, 2nd edition (Wiley, New York, 1992)

[2] H. Jing, L. Kuang, T. Fan, and X. Cao, "Measurement of large aspherical mirrors using coordinate measurement machine during the grinding process", Proc. SPIE 6148, 61480I-1-61480l-6(2005).

[3] S. Qian, P. Takacs, G. Sostero, and D. Cocco, "Portable long trace profiler: concept and solution", Rev. Sci. Instrum. 72, 3198-3204 (2001).

[4] J. R. P. Angel, and R. E. Parks, "Generation of off-axis aspherics", Proc. SPIE 332, 316-326 (1982).

[5] D. S. Anderson, R. E. Parks, and T. Shao, "A versatile profilometer for aspheric optics", in Proceedings of OFOT Workshop Technical Digest, Vol. 11, pp. 119-122. (Academic, Monterrey, CA 1990)

[6] D. S. Anderson, and J. H. Burge, "Swing arm Profilometry of Aspherics", Proc. SPIE 2356, 269-279 (1995).

[7] P. Su, C. J. Oh, R. E. Parks, and J. H. Burge, "Swing arm optical CMM for aspherics", Proc.SPIE 7426, 74260J-74260J -8 (2009).

[8] A. Lewis, S. Oldfield, M. Callender, A. Efstathiou, A. Gee, C. King, and D. Walker, "Accurate arm profilometry - traceable metrology for large mirrors", in Proceedings of Simposio de Metrología, pp. 101-105. (Academic, Mexico, 2006)

[9] A. Efstathiou, Design considerations for a hybrid swing arm profilometer to measure large aspheric optics (Ph.D thesis, London, 2007)

[10] A. Lewis, Uncertainty budget for the NPL-UCL swing arm profilometer operating in comparator mode (HMSO and Queen's printer, London, 2008).

[11] H. Jing, C. King, and D. Walker, "Simulation and validation of a prototype swing arm profilometer for measuring extremely large telescope mirror-segments", Opt. Express 18, 2036-2048 (2010).

[12] H. Jing, C. Lin , B. Fan, L. Kuang, S. Wu, and F. Wu, "Measurement of an Off-Axis Parabolic mirror using Coordinates Measurement Machine and Swing Arm Profilometer during the grinding process", (paper submitted to Optical Engineering) 